\title{
Charm and beauty of the Large Hadron Collider
}

\author{
Andrea Dainese $\ddagger$ \\ Università degli Studi di Padova and INFN, via Marzolo 8, 35131 Padova, Italy
}

\begin{abstract}
With the acceleration of lead nuclei in the LHC, heavy-ion physics will enter a new energy domain. One of the main novelties introduced by the 30 -fold energy-jump from RHIC to the LHC is the abundant heavy-quark production. After discussing a few examples of physics issues that can be addressed using heavy quarks, we present a selection of results on the expected experimental capability of ALICE, the dedicated heavy-ion experiment at the LHC, in the open-heavy-flavour sector.
\end{abstract}

PACS numbers: $25.75 .-\mathrm{q}, 14.65 . \mathrm{Dw}, 13.25 . \mathrm{Ft}$

\section{Introduction: novel aspects of heavy-ion physics at the LHC}

The nucleon-nucleon c.m.s. energy for $\mathrm{Pb}-\mathrm{Pb}$ collisions at the LHC, $\sqrt{s_{\mathrm{NN}}}=5.5 \mathrm{TeV}$, will exceed that available at RHIC by a factor about 30, opening up a new domain for the study of strongly-interacting matter in conditions of high temperature and energy density (QCD medium). Particle production at the LHC will present quantitative and qualitative new features, as discussed in the following.

Hard processes should contribute significantly to the total cross section. The mechanism of energy loss due to medium-induced gluon radiation allows to use the energetic partons produced in initial hard-scattering processes as probes to collect information on the opacity and density of the medium itself. At the LHC, the set of available probes will be extended both quantitatively and qualitatively. In fact, hard (light)quarks and gluons will be produced with high rates up to very large transverse momentum $\left(p_{\mathrm{t}}\right)$. Additionally, charm and beauty quarks, which, due to their masses, would show different attenuation patterns (see section 21), will become available for detailed measurements, since their production cross sections are expected to increase by factors 10 and 100, respectively, from RHIC to the LHC [1].

High-density parton distributions are expected to dominate particle production. The density of low momentum-fraction, $x$, gluons in the two colliding nuclei is expected to be close to saturation of the available phase space, so as to produce significant recombination effects. As an example, at central rapidity, low- $p_{\mathrm{t}} \mathrm{c}$ (b) quarks will be produced by partons with $x_{1} \simeq x_{2} \gtrsim 2 m_{\mathrm{c}}\left(m_{\mathrm{b}}\right) / \sqrt{s_{\mathrm{NN}}} \simeq 2.4(10) \mathrm{GeV} / 5500 \mathrm{GeV} \simeq$ $4(16) \times 10^{-4}$. In section 2 we will discuss how gluon fusions in this $x$ region are expected $\ddagger$ andrea.dainese@pd.infn.it 
Charm and beauty of the Large Hadron Collider

Table 1. Expected $\mathrm{Q} \overline{\mathrm{Q}}$ yields at the LHC, from NLO pQCD (parameters in the text) [5]. For $\mathrm{p}-\mathrm{Pb}$ and $\mathrm{Pb}-\mathrm{Pb}, \mathrm{PDF}$ shadowing is included and $N_{\text {coll }}$ scaling is applied.

\begin{tabular}{ccccc}
\hline colliding system & $\sqrt{s_{\mathrm{NN}}}$ & centrality & $N^{\mathrm{c} \overline{\mathrm{c}}} /$ event & $N^{\mathrm{b} \overline{\mathrm{b}}} /$ event \\
\hline $\mathrm{pp}$ & $14 \mathrm{TeV}$ & minimum bias & 0.16 & 0.0072 \\
$\mathrm{p}-\mathrm{Pb}$ & $8.8 \mathrm{TeV}$ & minimum bias & 0.78 & 0.029 \\
$\mathrm{~Pb}-\mathrm{Pb}$ & $5.5 \mathrm{TeV}$ & central $\left(0-5 \% \sigma^{\text {tot }}\right)$ & 115 & 4.6 \\
\hline
\end{tabular}

to affect $\mathrm{c} \overline{\mathrm{c}}$ (and, to a lower extent, $\mathrm{b} \overline{\mathrm{b}}$ ) production, not only in $\mathrm{p}-\mathrm{Pb}$ and $\mathrm{Pb}-\mathrm{Pb}$ collisions (nuclear shadowing), but possibly even in pp collisions.

\section{Heavy-quark phenomenology from pp to nucleus-nucleus collisions}

Heavy-quark pairs, $\mathrm{Q} \overline{\mathrm{Q}}$, are produced in partonic scatterings with large virtuality (momentum transfer) $Q \gtrsim 2 m_{\mathrm{Q}}$. Therefore, the production cross sections in nucleon-nucleon (NN) collisions can be calculated in the framework of collinearly factorized perturbative QCD (pQCD). The differential Q $\bar{Q}$ cross section is written as:

$$
\begin{aligned}
\mathrm{d} \sigma^{\mathrm{NN} \rightarrow \mathrm{Q} \overline{\mathrm{Q}} X}\left(\sqrt{s_{\mathrm{NN}}}, m_{\mathrm{Q}}, \mu_{\mathrm{F}}^{2}, \mu_{\mathrm{R}}^{2}\right)= & \sum_{i, j=\mathrm{q}, \overline{\mathrm{q}}, \mathrm{g}} f_{i}\left(x_{1}, \mu_{\mathrm{F}}^{2}\right) \otimes f_{j}\left(x_{2}, \mu_{\mathrm{F}}^{2}\right) \otimes \\
& \mathrm{d} \hat{\sigma}^{i j \rightarrow \mathrm{Q} \overline{\mathrm{Q}}\{k\}}\left(\alpha_{\mathrm{s}}\left(\mu_{\mathrm{R}}^{2}\right), \mu_{\mathrm{F}}^{2}, m_{\mathrm{Q}}, x_{1} x_{2} s_{\mathrm{NN}}\right),
\end{aligned}
$$

where the partonic $\mathrm{d} \hat{\sigma}^{i j \rightarrow \mathrm{Q}} \overline{\mathrm{Q}}\{k\}$ is calculable as a power series in the strong coupling $\alpha_{\mathrm{s}}$, which depends on the renormalization scale $\mu_{\mathrm{R}}$; currently, calculations are performed up to next-to-leading order $(\mathrm{NLO}), \mathcal{O}\left(\alpha_{\mathrm{s}}^{3}\right)$. The nucleon Parton Distribution Function (PDF) for the parton of type $i$ at momentum fraction $x_{1}$ and factorization scale $\mu_{\mathrm{F}}$, which can be interpreted as the virtuality of the hard process, is denoted by $f_{i}\left(x_{1}, \mu_{\mathrm{F}}^{2}\right)$.

The expected yields in pp collisions at $\sqrt{s}=14 \mathrm{TeV}$ are reported in the first line of Table 1. These numbers are obtained at NLO using the MNR program [2] with $m_{\mathrm{c}}=1.2 \mathrm{GeV}$ and $\mu_{F}=\mu_{R}=2 m_{\mathrm{c}}$ for charm and $m_{\mathrm{b}}=4.75 \mathrm{GeV}$ and $\mu_{F}=\mu_{R}=m_{\mathrm{b}}$ for beauty; the PDF set is CTEQ 4M [3]. The predicted yields have large uncertainties, of about a factor 2, estimated by varying the values of the masses and of the scales (much smaller uncertainties, $\approx 20 \%$, arise from the indetermination in the PDFs) [4,5]. The theoretical uncertainty band for the D-meson cross section as a function of $p_{\mathrm{t}}$ will be shown in section 3, along with the expected sensitivity of the ALICE experiment [6].

As aforementioned, in the $x$ range relevant for $\mathrm{Q} \overline{\mathrm{Q}}$ production, the PDFs will be close to phase-space saturation and, already in the case of pp collisions, there will be important gluon-fusion effects ( $g g \rightarrow \mathrm{g}$ ). These can be accounted for in the PDF scaleevolution equations by adding to the standard linear DGLAP term a negative nonlinear (quadratic) term (see [7] and references therein):

$$
\partial f_{\mathrm{g}}\left(x, Q^{2}\right) / \partial \log Q^{2}=\left[\text { DGLAP term of } \mathcal{O}\left(f_{\mathrm{g}}\right)\right]-\left[\text { term of } \mathcal{O}\left(f_{\mathrm{g}}^{2}\right)\right] .
$$



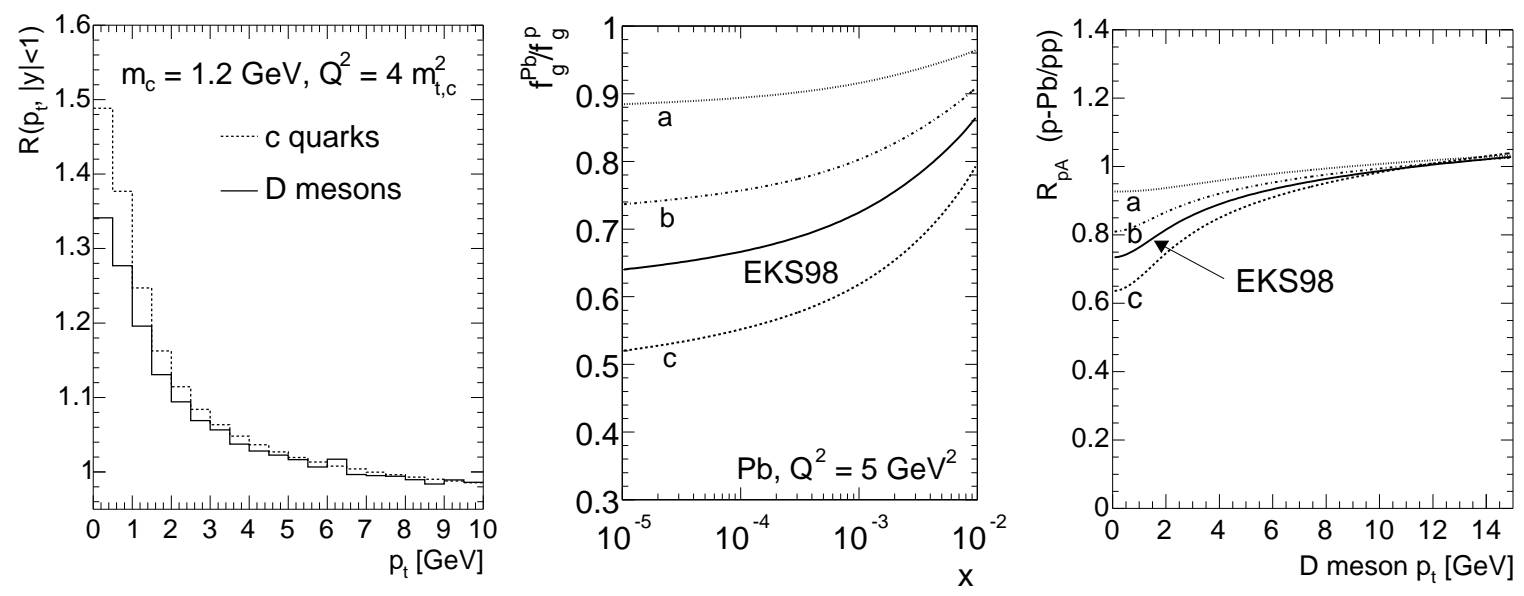

Figure 1. Left: enhancement due to nonlinear gluon evolution for c quarks and D mesons in pp collisions at $\sqrt{s}=14 \mathrm{TeV}$ [9]. Centre: modification of the gluon PDF in a $\mathrm{Pb}$ nucleus at $Q^{2} \simeq 4 m_{\mathrm{c}}^{2}$. Right: corresponding $R_{\mathrm{pA}}^{\mathrm{D}}$ in $\mathrm{p}-\mathrm{Pb}$ at $\sqrt{s_{\mathrm{NN}}}=8.8 \mathrm{TeV}$.

The nonlinear term, currently calculated only at LO, 'slows down' the $Q^{2}$ evolution at given $x$. It has been shown [7] that, for $x \lesssim 10^{-2}$, it allows to have a higher gluon density at small $Q^{2}\left(\lesssim 10 \mathrm{GeV}^{2}\right.$ ), with respect to that obtained with DGLAP terms only, and to maintain at the same time a good fit of the proton structure function data from HERA. A higher gluon PDF would imply an enhancement, w.r.t. to DGLAP-based calculations, of $c \bar{c}$ production at low $p_{\mathrm{t}}$ at LHC energy [8]. Figure 1 (left) shows, as a function of $p_{\mathrm{t}}$, the enhancement at the c-quark and at the D-meson level, for $m_{\mathrm{c}}=1.2 \mathrm{GeV}$ and $\mu_{\mathrm{F}}^{2}=\mu_{\mathrm{R}}^{2}=Q^{2}=4 m_{\mathrm{t}, \mathrm{c}}^{2} \equiv 4\left(m_{\mathrm{c}}^{2}+p_{\mathrm{t}}^{2}\right)[9]$. Here, as well as for the other results presented in the following, the hadronization of heavy quarks is performed using the string fragmentation model implemented in PYTHIA [10]. The enhancement survives fragmentation and it is of about $30 \%$ for D-meson $p_{\mathrm{t}} \rightarrow 0$, even in this 'pessimistic' case where relatively-large $Q^{2}$ values are considered (it should be noted, however, that this is a $\mathrm{LO}$ result and the effect might be smaller at NLO). In section 3 we will discuss a possible strategy to detect the enhancement in pp collisions at the LHC.

For hard processes, in the absence of nuclear and medium effects, a nucleus-nucleus (or p-nucleus) collision would behave as a superposition of independent NN collisions. The charm and beauty differential yields would then scale from pp to AA (or pA) proportionally to the number $N_{\text {coll }}$ of inelastic NN collisions (binary scaling):

$$
\mathrm{d}^{2} N_{\mathrm{AA}(\mathrm{pA})}^{\mathrm{Q}} / \mathrm{d} p_{\mathrm{t}} \mathrm{d} y=N_{\text {coll }} \times \mathrm{d}^{2} N_{\mathrm{pp}}^{\mathrm{Q}} / \mathrm{d} p_{\mathrm{t}} \mathrm{d} y .
$$

Binary scaling is, indeed, expected to break down due to both initial-state effects, such as nuclear shadowing of the PDFs, and final-state effects, such as parton energy loss in the medium formed in nucleus-nucleus collisions. As a consequence, as we will detail in the following, heavy quarks are important tools to probe and investigate these effects.

In Table 1 we report the $c \overline{\mathrm{c}}$ and $\mathrm{b} \overline{\mathrm{b}}$ yields in $\mathrm{p}-\mathrm{Pb}$ and $\mathrm{Pb}-\mathrm{Pb}$ collisions calculated including in the NLO pQCD calculation the EKS98 parameterization [11] of the PDFs 


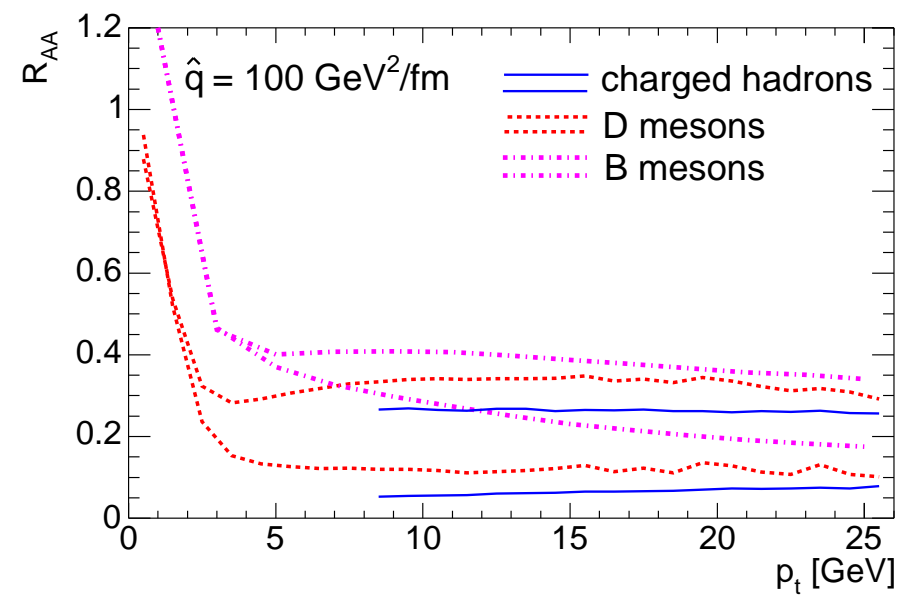

Figure 2. Nuclear modification factors for charged hadrons [15], D mesons $\left(m_{\mathrm{c}}=1.2 \mathrm{GeV}\right)$ and $\mathrm{B}$ mesons $\left(m_{\mathrm{b}}=4.8 \mathrm{GeV}\right)[16]$ in central $(0-$ $\left.10 \% \sigma^{\text {tot }}\right) \mathrm{Pb}-\mathrm{Pb}$ collisions relative to binary scaling from pp collisions, at $\sqrt{s_{\mathrm{NN}}}=5.5 \mathrm{TeV}$. The D-meson result does not include feed-down from $\mathrm{B} \rightarrow \mathrm{D}+X$ decays, which is, however, expected to be rather small $(\sim 5 \%)[18]$.

nuclear modification $f_{i}^{\mathrm{Pb}}\left(x, Q^{2}\right) / f_{i}^{\mathrm{p}}\left(x, Q^{2}\right)$, shown in Fig. 1] (centre) for $Q^{2}=5 \mathrm{GeV}^{2}$, and applying binary scaling [5]. The charm (beauty) cross-section reduction induced by shadowing is about $35 \%(20 \%)$ in $\mathrm{Pb}-\mathrm{Pb}$ and $15 \%(10 \%)$ in $\mathrm{p}-\mathrm{Pb}$. There is a significant uncertainty on the strength of shadowing in the small- $x$ region and some models predict much larger suppression than EKS98 (see [12] for a review). The comparison of $Q \bar{Q}$ production in pp and p-Pb collisions (where final-state effects are not present) is regarded as a sensitive tool to probe nuclear PDFs at the LHC. The ratio of invariant-mass spectra of di-leptons from heavy-quark decays in $\mathrm{p}-\mathrm{Pb}$ and pp collisions would measure the nuclear modification $f_{\mathrm{g}}^{\mathrm{Pb}} / f_{\mathrm{g}}^{\mathrm{p}}[12]$. Another promising observable in this respect is the nuclear modification factor of the D-meson $p_{\mathrm{t}}$ distribution:

$$
R_{\mathrm{pA}(\mathrm{AA})}^{\mathrm{D}}\left(p_{\mathrm{t}}\right)=\frac{1}{N_{\text {coll }}} \times \frac{\mathrm{d}^{2} N_{\mathrm{pA}(\mathrm{AA})}^{\mathrm{D}} / \mathrm{d} p_{\mathrm{t}} \mathrm{d} y}{\mathrm{~d}^{2} N_{\mathrm{pp}}^{\mathrm{D}} / \mathrm{d} p_{\mathrm{t}} \mathrm{d} y} .
$$

We note that at the $\mathrm{LHC}$, the $\mathrm{pp}, \mathrm{p}-\mathrm{Pb}$ and $\mathrm{Pb}-\mathrm{Pb}$ runs will have different $\sqrt{s_{\mathrm{NN}}}$ values; however, pQCD can be used to extrapolate the measured cross sections between different energies [4] and, thus, calculate the $R_{\mathrm{pA}(\mathrm{AA})}$ ratios. In Fig. 1 (right) we show the sensitivity of $R_{\mathrm{pA}}^{\mathrm{D}}$ to different shadowing scenarios, obtained by varying the modification of the PDFs (shown for gluons in the central panel of the same figure).

Experiments at RHIC have shown that the nuclear modification factor $R_{\mathrm{AA}}$ is an effective tool for the study of the interaction of the hard partons with the medium produced in nucleus-nucleus collisions. Heavy-quark medium-induced quenching is one of the most captivating topics to be addressed in $\mathrm{Pb}-\mathrm{Pb}$ collisions at the $\mathrm{LHC}$, where both $\mathrm{c}$ and b quarks will be produced with high rates (see Table 1). Due to the QCD nature of parton energy loss, quarks are predicted to lose less energy than gluons (that have a higher colour charge) and, in addition, the 'dead-cone effect' is expected to reduce the energy loss of massive quarks $[13,14]$. Therefore, one should observe a pattern of gradually decreasing $R_{\mathrm{AA}}$ suppression when going from gluon-originated light-flavour hadrons $(h)$ to $\mathrm{D}$ and to $\mathrm{B}$ mesons: $R_{\mathrm{AA}}^{h} \lesssim R_{\mathrm{AA}}^{\mathrm{D}} \lesssim R_{\mathrm{AA}}^{\mathrm{B}}$. In Fig. 2 we report recent estimates of these quantities for central $\mathrm{Pb}-\mathrm{Pb}$ collisions at the $\mathrm{LHC}[15,16]$, obtained in the framework of a model [15] where energy loss is simulated in a parton-by-parton 
approach combining the BDMPS 'quenching weights' [17] and a Glauber-model-based definition of the in-medium parton path length. For $\mathrm{c}$ and $\mathrm{b}$ quarks, the quenching weights were specifically calculated using the formalism developed in [14]. The BDMPS transport coefficient (a measure of the medium density) at the LHC was set to the value $\hat{q}=100 \mathrm{GeV}^{2} / \mathrm{fm}$, estimated on the basis of the analysis of RHIC data performed in [15]. The results are plotted as bands that represent the theoretical uncertainty $[15,16]$. For charged hadrons, $R_{\mathrm{AA}}$ is given for $p_{\mathrm{t}} \gtrsim 8 \mathrm{GeV}$ because at lower $p_{\mathrm{t}}$ the soft-particle component produced from the radiated gluons, which is not implemented in the model, might strongly contribute in shaping the nuclear modification factor. Whereas for D and $\mathrm{B}$ mesons $R_{\mathrm{AA}}$ can be calculated down to $p_{\mathrm{t}}=0$ by assigning a 'thermal' transverse momentum (according to $\mathrm{d} N / \mathrm{d} m_{\mathrm{t}} \propto m_{\mathrm{t}} \exp \left(-m_{t} / T\right), T=300 \mathrm{MeV}$ ) to the $\mathrm{c}$ and $\mathrm{b}$ quarks that lose most of their initial energy in the medium [16]. The presence of this thermalized component determines the rise of $R_{\mathrm{AA}}^{\mathrm{D}, \mathrm{B}}$ at $p_{\mathrm{t}} \rightarrow 0$. It should be mentioned here that, in the low $p_{\mathrm{t}}$ region, the hadronization of heavy quarks in nucleusnucleus collisions is likely to happen inside the medium via parton recombination [19], thus producing deviations from the simple pattern obtained from the aforementioned thermalization assumption.

\section{Looking for heavy quarks at the LHC: tools, techniques, performance}

Three experiments will participate in the LHC heavy-ion program: ALICE, the dedicated heavy-ion experiment [6]; CMS, with a strong heavy-ion program [20]; (most probably) ATLAS, which recently expressed interest in participating [21]. The three detectors have different features and design requirements, but all of them are expected to have excellent capabilities for heavy-flavour measurements. Their complementarity will provide a very broad coverage in terms of phase-space, decay channels and observables.

Experimentally, the two key elements for a rich heavy-flavour program are: tracking/vertexing and particle identification (PID).

Open charm and beauty mesons have typical life-times of few hundred microns ( $c \tau$ values are about $125-300 \mu \mathrm{m}$ for D mesons and $500 \mu \mathrm{m}$ for B mesons) and the most direct detection strategy is the identification of single tracks or vertices that are displaced from the interaction vertex. The detector capability to perform this task is determined by the impact parameter $\S\left(d_{0}\right)$ resolution. All experiments will be equipped with high position-resolution silicon-detector layers, including pixels, for precise tracking and impact parameter measurement also in the high-multiplicity environment of central $\mathrm{Pb}-\mathrm{Pb}$ collisions. Tracking is done in the central (pseudo)rapidity region: $|\eta|<0.9$ for ALICE and $|\eta| \lesssim 1.5$ for CMS and ATLAS. In Fig. 3 we show the $d_{0}$ resolution of ALICE and CMS\|, along with the $p_{\mathrm{t}}$ resolution, which is another important ingredient for heavy-flavour measurements (e.g. it determines the invariant mass resolution). The

$\S$ We define as impact parameter the distance of closest approach to the interaction vertex of the track projection in the plane transverse to the beam direction.

\| ATLAS may perform similarly to ALICE, but systematic $\mathrm{Pb}-\mathrm{Pb}$ studies are still in progress. 

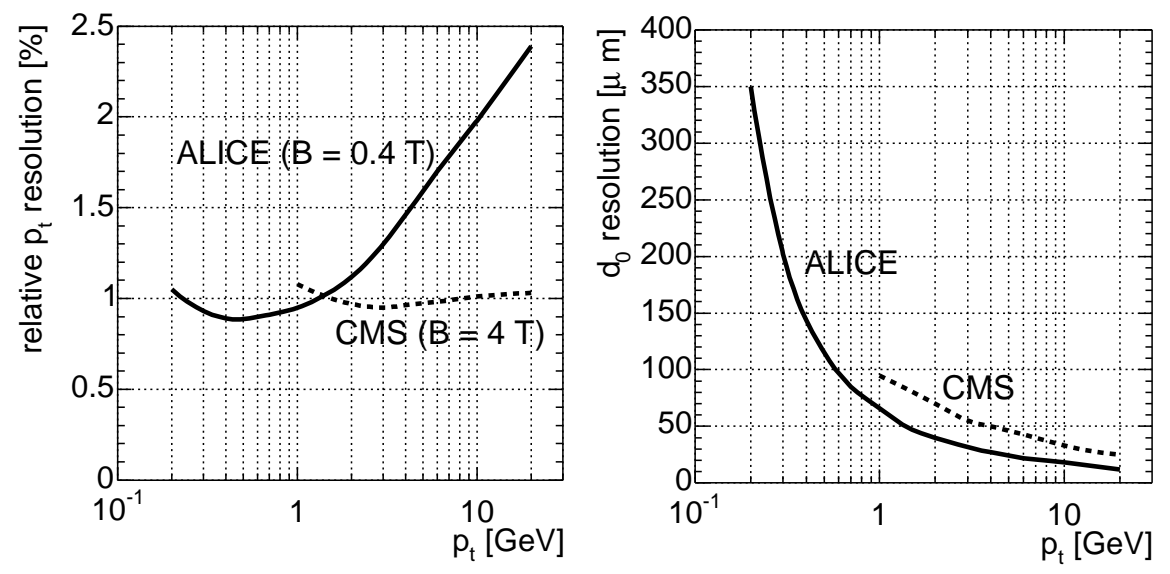

Figure 3. Transverse momentum (left) and transverse track impact parameter (right) resolutions for the ALICE and CMS detectors in $\mathrm{Pb}-\mathrm{Pb}$ collisions.

main difference between the two experiments is given by the magnetic field values: ALICE $(0.4 \mathrm{~T})$ has a very low $p_{\mathrm{t}}$ cutoff of $0.2 \mathrm{GeV}$, while CMS $(4 \mathrm{~T})$ has a higher cutoff of $1 \mathrm{GeV}$ but better $p_{\mathrm{t}}$ resolution at high $p_{\mathrm{t}}$. The $d_{0}$ resolutions are quite similar and better than $50 \mu \mathrm{m}$ for $p_{\mathrm{t}} \gtrsim 1.5-3 \mathrm{GeV}$.

Both lepton and hadron identification are important for heavy-flavour detection. D and $\mathrm{B}$ mesons have relatively-large branching ratios (BR) in the semi-leptonic channels, $\simeq 10 \%$ to electrons and $\simeq 10 \%$ to muons, and inclusive cross-section measurements can be performed via single leptons or di-leptons. ALICE can identify electrons with $p_{\mathrm{t}}>1 \mathrm{GeV}$ and $|\eta|<0.9$, via transition radiation and $\mathrm{d} E / \mathrm{d} x$ measurements, and muons in the forward region, $2.5<\eta<4$, which allows a very low $p_{\mathrm{t}}$ cutoff of $1 \mathrm{GeV}$. CMS and ATLAS have a broad pseudorapidity coverage for muons, $|\eta|<2.4$ and $|\eta|<2.7$, respectively, but they have a quite-high $p_{\mathrm{t}}$ cutoff of $\approx 4 \mathrm{GeV}$. Both CMS and ATLAS have high-resolution electro-magnetic calorimeters that can be used to identify electrons, although performance studies for heavy-ion collisions have not been carried out yet. Semi-leptonic inclusive measurements do not provide direct information on the $\mathrm{D}(\mathrm{B})$-meson $p_{\mathrm{t}}$ distribution, especially at low $p_{\mathrm{t}}$, because of the weak correlation between the lepton and meson momenta. Therefore, for charm in particular, the reconstruction of exclusive (hadronic) decays is preferable. In this case, in a high-multiplicity environment, hadron identification allows a more effective rejection of the large combinatorial background in the low- $p_{\mathrm{t}}$ region. ALICE disposes of $\pi / \mathrm{K} / \mathrm{p}$ separation via $\mathrm{d} E / \mathrm{d} x$ and time-of-flight measurement for $p<3-4 \mathrm{GeV}$ and $|\eta|<0.9$.

In the following we present results on the expected performance for the detection of $\mathrm{D}$ and B mesons in ALICE (CMS and ATLAS studies are described in $[4,22]$ ). The $c \overline{\mathrm{C}}$ and $\mathrm{b} \overline{\mathrm{b}}$ cross sections from Table 1 are used and the charged-particles rapidity density of a central $\left(0-5 \% \sigma^{\text {tot }}\right) \mathrm{Pb}-\mathrm{Pb}$ collision is assumed to be $\mathrm{d} N_{\mathrm{ch}} / \mathrm{d} y \simeq 6000$. The results, based on realistic detector simulations, are given for samples of $10^{9} \mathrm{pp}$ events (about 9 months of data taking) and of $10^{7}$ central $\mathrm{Pb}-\mathrm{Pb}$ events (about 1 month of data taking). 
Charm reconstruction in ALICE. One of the most promising channels for open charm detection is the $\mathrm{D}^{0} \rightarrow \mathrm{K}^{-} \pi^{+}$decay (and charge conjugate) that has a $\mathrm{BR}$ of $3.8 \%$. The expected yields $(\mathrm{BR} \times \mathrm{d} N / \mathrm{d} y$ at $y=0)$, in central $\mathrm{Pb}-\mathrm{Pb}\left(0-5 \% \sigma^{\text {tot }}\right)$ at $\sqrt{s_{\mathrm{NN}}}=5.5 \mathrm{TeV}$ and in pp collisions at $\sqrt{s}=14 \mathrm{TeV}$ are $5.3 \times 10^{-1}$ and $7.5 \times 10^{-4}$ per event, respectively.

The main feature of this decay topology is the presence of two tracks with impact parameters $d_{0} \sim 100 \mu \mathrm{m}$. The detection strategy [23] to cope with the large combinatorial background from the underlying event is based on the selection of displaced-vertex topologies, i.e. two tracks with large impact parameters and good alignment between the $\mathrm{D}^{0}$ momentum and flight-line, and on invariant-mass analysis to extract the signal yield. This strategy was optimized separately for $\mathrm{pp}$ and $\mathrm{Pb}-\mathrm{Pb}$ collisions, as a function of the $\mathrm{D}^{0}$ transverse momentum, and statistical and systematic errors were estimated [18].

Figure 4 (left) shows the expected sensitivity of ALICE for the measurement of the $\mathrm{D}^{0} p_{\mathrm{t}}$-differential cross section in pp collisions, compared to the pQCD calculations uncertainty that we mentioned in section 2, and in central $\left(0-5 \% \sigma^{\text {tot }}\right) \mathrm{Pb}-\mathrm{Pb}$ collisions. The low- $p_{\mathrm{t}}$ reach, provided by the moderate ALICE magnetic field $(0.4 \mathrm{~T})$ and the $\mathrm{K} / \pi$ separation via time-of-flight, allows to address the issue of charm 'enhancement' in pp collisions due to nonlinear gluon evolution (section 2). Here, the idea is that the effect - enhancement only at low $p_{\mathrm{t}}$ — cannot be mimicked by standard DGLAP-based pQCD just by varying the input parameters. This is illustrated in Fig. 4 (from [9]), where the data-to-theory ratio is plotted versus the $\mathrm{D}^{0} p_{\mathrm{t}}$. The data are obtained with $m_{\mathrm{c}}=1.2 \mathrm{GeV}$ and $Q^{2}=4 m_{\mathrm{t}, \mathrm{c}}^{2}$ and they include the enhancement (from Fig. [1 (left)), while the theory results do not: only for $m_{\mathrm{c}} \lesssim 1.1 \mathrm{GeV}$ the theory can mimic the enhancement, but such small values are not supported by lower- $\sqrt{s}$ measurements.

The direct measurement of the D-meson $p_{\mathrm{t}}$ distribution allows a good sensitivity to the suppression of the nuclear modification factor due to c-quark energy loss in $\mathrm{Pb}-\mathrm{Pb}$ collisions. Figure 5 (left) shows the estimated experimental errors on $R_{\mathrm{AA}}^{\mathrm{D}}$, reported on a curve that lies in the middle of the D-meson band from Fig. 2 ,

Simulation studies to assess the performance for $\mathrm{D}^{0}$ reconstruction in $\mathrm{p}-\mathrm{Pb}$ are in progress and the results are expected to be similar to the pp case [18]. This might allow to measure nuclear shadowing via the $R_{\mathrm{pA}}^{\mathrm{D}}$ ratio, as discussed in section 2

Beauty via single electrons in ALICE. The expected yield $(\mathrm{BR} \times \mathrm{d} N / \mathrm{d} y$ at $y=0)$ for $\mathrm{B} \rightarrow e^{ \pm}+X$ per central $\left(0-5 \% \sigma^{\text {tot }}\right) \mathrm{Pb}-\mathrm{Pb}$ collision at $\sqrt{s_{\mathrm{NN}}}=5.5 \mathrm{TeV}$ is $9 \times 10^{-2}$.

The main sources of background electrons are: (a) decays of D mesons; (b) decays of light mesons (e.g. $\rho$ and $\omega$ ); (c) conversions of photons in the beam pipe or in the inner detector layers and (d) pions misidentified as electrons. Given that electrons from beauty have average impact parameter $d_{0} \simeq 500 \mu \mathrm{m}$ and a hard momentum spectrum, it is possible to obtain a high-purity sample with a strategy that relies on: electron identification with a combined $\mathrm{d} E / \mathrm{d} x$ and transition radiation selection, which allows to reduce the pion contamination by a factor $10^{4}$; impact parameter cut to reject misidentified pions and electrons from sources (b) and (c); transverse momentum cut to 

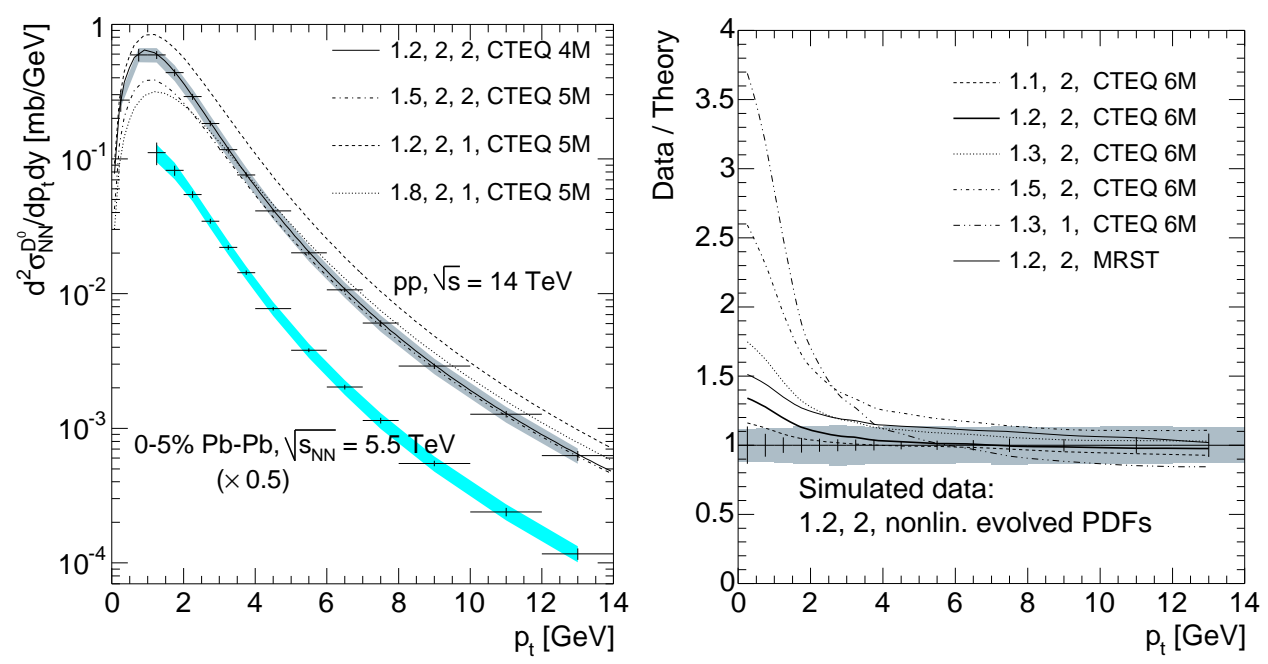

Figure 4. Left: $\mathrm{D}^{0}$ production cross section vs. $p_{\mathrm{t}}$, as it can be measured by ALICE in pp $\left(10^{9}\right.$ events $)$ and in central $\mathrm{Pb}-\mathrm{Pb}\left(10^{7}\right.$ events $)$ collisions; statistical (bars) and $p_{\mathrm{t}}$-dependent systematic errors (band) are shown; a normalization error of $5 \%$ in pp and $11 \%$ in $\mathrm{Pb}-\mathrm{Pb}$ is not shown; pQCD predictions for different sets of parameters $\left(m_{\mathrm{c}}[\mathrm{GeV}], \mu_{F} / m_{\mathrm{t}, \mathrm{c}}, \mu_{R} / m_{\mathrm{t}, \mathrm{c}}\right.$, PDF set) are also reported for the pp case. Right: ratios of simulated ALICE $\mathrm{D}^{0}$ data to pQCD curves (parameters: $m_{\mathrm{c}}[\mathrm{GeV}]$, $Q / m_{\mathrm{t}, \mathrm{c}}=\mu_{F} / m_{\mathrm{t}, \mathrm{c}}=\mu_{R} / m_{\mathrm{t}, \mathrm{c}}$, PDF set); the data contain the enhancement due to nonlinear gluon evolution while the theory curves do not [9].
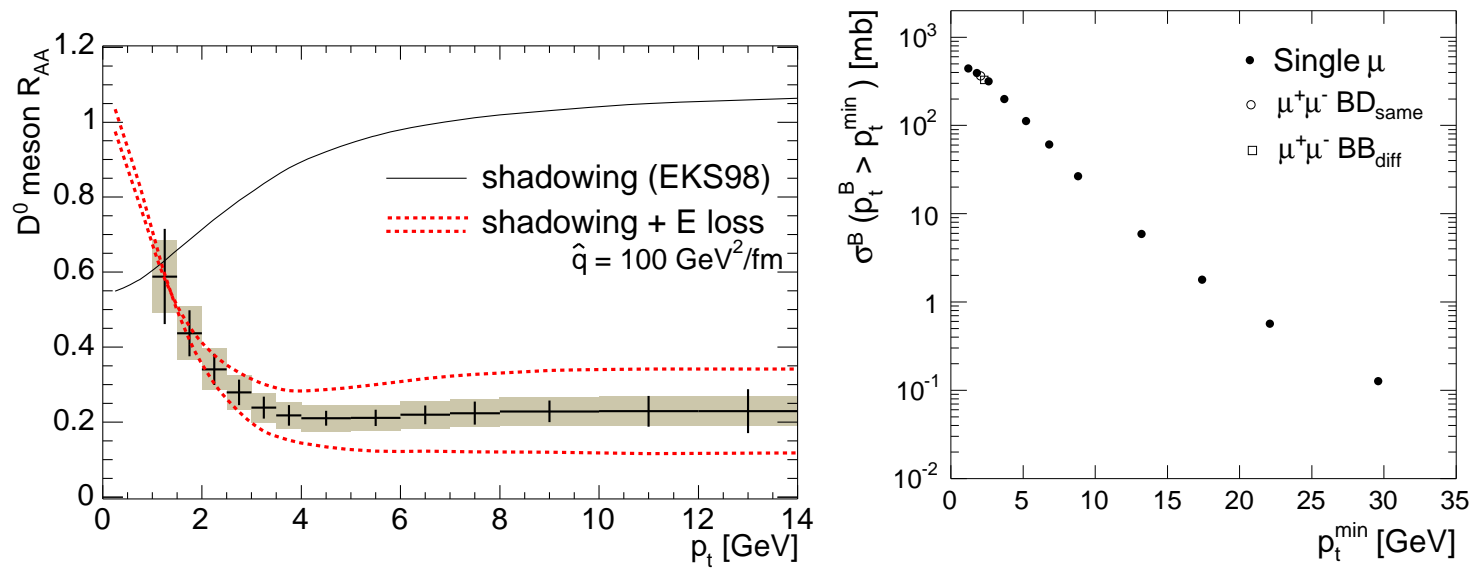

Figure 5. Left: ALICE sensitivity for $\mathrm{D}^{0}$-meson $R_{\mathrm{AA}}$ with $10^{7}$ central $\mathrm{Pb}-\mathrm{Pb}$ events and $10^{9}$ pp events; statistical (bars) and systematic (bands) errors are shown. Right: B production cross section vs. $p_{\mathrm{t}}^{\text {min }}$ reconstructed by ALICE with single muons and di-muons in $10^{7}$ central $\mathrm{Pb}-\mathrm{Pb}$ collisions; only (very small) statistical errors shown.

reject electrons from charm decays. As an example, with $d_{0}>180 \mu \mathrm{m}$ and $p_{\mathrm{t}}>2 \mathrm{GeV}$, the expected statistics of electrons from $\mathrm{B}$ decays is $5 \times 10^{4}$ for $10^{7}$ central $\mathrm{Pb}-\mathrm{Pb}$ events, with a contamination of about $10 \%$, mainly given by electrons from charm decays [4,24].

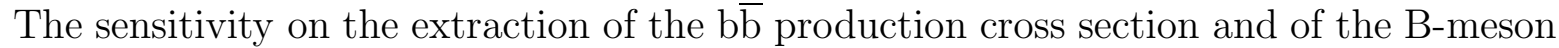
$p_{\mathrm{t}}$ distribution is currently being investigated. 
Beauty via (di-)muons in ALICE. B production in $\mathrm{Pb}-\mathrm{Pb}$ collisions can be measured also in the ALICE forward muon spectrometer, $2.5<\eta<4$, analyzing the single-muon $p_{\mathrm{t}}$ distribution and the opposite-sign di-muons invariant mass distribution [24].

The main backgrounds to the 'beauty muon' signal are $\pi^{ \pm}, \mathrm{K}^{ \pm}$and charm decays. The cut $p_{\mathrm{t}}>1.5 \mathrm{GeV}$ is applied to all reconstructed muons in order to increase the signal-to-background ratio. For the opposite-sign di-muons, the residual combinatorial background is subtracted using the technique of event-mixing and the resulting distribution is subdivided into two samples: the low-mass region, $M_{\mu^{+} \mu^{-}}<5 \mathrm{GeV}$, dominated by muons originating from a single b quark decay through $\mathrm{b} \rightarrow \mathrm{c}\left(\rightarrow \mu^{+}\right) \mu^{-}$ $\left(\mathrm{BD}_{\text {same }}\right)$, and the high-mass region, $5<M_{\mu^{+} \mu^{-}}<20 \mathrm{GeV}$, dominated by $\mathrm{b} \overline{\mathrm{b}} \rightarrow \mu^{+} \mu^{-}$, with each muon coming from a different quark in the pair $\left(\mathrm{BB}_{\text {diff }}\right)$. Both samples have a background from $c \overline{\mathrm{c}} \rightarrow \mu^{+} \mu^{-}$and a fit is done to extract the charm- and beautycomponent yields. The single-muon $p_{\mathrm{t}}$ distribution has three components with different slopes: $\mathrm{K}$ and $\pi$, charm, and beauty decays. Also in this case a fit technique allows to extract a $p_{\mathrm{t}}$ distribution of muons from $\mathrm{B}$ decays. From the $\mu$-level cross sections a Monte-Carlo-based procedure is used to compute B-level cross sections for the data sets (low-mass $\mu^{+} \mu^{-}$, high-mass $\mu^{+} \mu^{-}$, and $p_{\mathrm{t}}$-binned single-muon distribution), each set covering a specific B-meson $p_{\mathrm{t}}>p_{\mathrm{t}}^{\text {min }}$ region, as preliminarly shown in Fig. 5 (right). Since only minimal cuts are applied, the reported statistical errors are very small and high- $p_{\mathrm{t}}$ reach is excellent. Systematic errors are currently under study.

\section{Summary}

We have discussed how heavy quarks, abundantly produced at LHC energies, will allow to address several physics issues, in proton-proton, proton-nucleus and nucleus-nucleus collisions. In particular, they provide tools to:

- probe, via parton energy loss and its predicted mass dependence, the high-density $\mathrm{QCD}$ medium formed in $\mathrm{Pb}-\mathrm{Pb}$ collisions;

- probe, in pp collisions, the pQCD calculations parameters space;

- probe the small- $x$ regime of the PDFs, where saturation/recombination effects are expected to be important, even in pp collisions.

The excellent tracking, vertexing and particle identification performance of ALICE, ATLAS and CMS will allow to fully explore this rich phenomenology, as we have shown with some specific ALICE studies on D and B meson measurements.

Acknowledgment. The author, member of the ALICE Collaboration, would like to thank the ALICE off-line group, within which part of the results here reported have been obtained. Fruitful discussions on the manuscript with F. Antinori are also acknowledged.

[1] Vogt $\mathrm{R}$ these proceedings

[2] Mangano M, Nason P and Ridolfi G 1992 Nucl. Phys. B 373295

[3] Lai H L et al. 1997 Phys. Rev. D 551280 
[4] Bedjidian M et al 2003 CERN Yellow Report in press Preprint hep-ph/0311048

[5] Carrer N and Dainese A 2003 ALICE-INT-2003-019 Preprint hep-ph/0311225

[6] ALICE Coll. 2004 Physics Performance Report Vol. I J. Phys. G 301517 (CERN/LHCC 2003-049)

[7] Eskola K J, Honkanen H, Kolhinen V J, Qiu J w and Salgado C A 2004 Nucl. Phys. B 660211

[8] Eskola K J, Kolhinen V J and Vogt R 2004 Phys. Lett. B 582157

[9] Dainese A, Vogt R, Bondila M, Eskola K J and Kolhinen V J 2004 J. Phys. G 301787

[10] Sjöstrand T et al. 2001 Computer Phys. Commun. 135238

[11] Eskola K J, Kolhinen V J and Salgado C A 1999 Eur. Phys. J. C 961

[12] Accardi A et al 2003 CERN Yellow Report in press Preprint hep-ph/0308248

[13] Dokshitzer Yu L and Kharzeev D E 2001 Phys. Lett. B 519199

[14] Armesto N, Salgado C A and Wiedemann U A 2004 Phys. Rev. D 69114003

[15] Dainese A, Loizides C and Paic G 2005 Eur. Phys. J. C 38461

[16] Armesto N, Dainese A, Salgado C A and Wiedemann U A Preprint hep-ph/0501225

[17] Salgado C A and Wiedemann U A 2003 Phys. Rev. D 68014008

[18] Dainese A 2003 Ph.D. Thesis Università degli Studi di Padova Preprint nucl-ex/0311004

[19] Molnar D these proceedings

[20] CMS Coll. Heavy-Ion Group http://cmsdoc.cern.ch/cms/PRS/hi/

[21] ATLAS Coll. 2004 Heavy-ion Letter of Intent (CERN/LHCC 2004-009)

[22] Petrushanko S these proceedings; Klay J these proceedings

[23] Carrer N, Dainese A and Turrisi R 2003 J. Phys. G 29575

[24] ALICE Coll. Physics Performance Report Vol. II in preparation 